\title{
Intertextualité et histoire naturelle
}

Étude génétique du chapitre « Fécondité » de La Mer de Michelet

\section{Élisabeth Plas}

\section{(2) OpenEdition}

Journals

Édition électronique

URL : http://journals.openedition.org/genesis/981

DOI : $10.4000 /$ genesis. 981

ISSN : 2268-1590

Éditeur :

Presses universitaires de Paris Sorbonne (PUPS), Société internationale de génétique artistique littéraire et scientifique (SIGALES)

\section{Édition imprimée}

Date de publication : 10 avril 2012

Pagination : 187-203

ISBN : 978-2-84050-822-9

ISSN : $1167-5101$

\section{Référence électronique}

Élisabeth Plas, «Intertextualité et histoire naturelle », Genesis [En ligne], 34 | 2012, mis en ligne le 10 avril 2014, consulté le 14 juin 2020. URL : http://journals.openedition.org/genesis/981 ; DOI : https:// doi.org/10.4000/genesis.981 


\title{
Intertextualité et histoire naturelle : étude génétique du chapitre «Fécondité » de La Mer de Michelet
}

\author{
Élisabeth Plas
}

On pense souvent que la génétique textuelle est une pratique ésotérique réservée aux chercheurs aguerris. Il s'agit certes d'une discipline exigeante, mais (comme le montraient déjà les travaux pédagogiques publiés dans notre numéro 16) le contact avec les manuscrits peut être source d'inspiration pour les étudiants talentueux et motivés. Nous publions ici, sans retouches, un travail de validation rendu à l'issue d'un séminaire d'initiation à la critique génétique.

$\mathrm{L}$ es manuscrits du chapitre « Fécondité1 » de l'ouvrage de Michelet intitulé La Mer, troisième volet publié en 1861 de son cycle naturaliste commencé avec L'Oiseau en 1856 , et poursuivi en 1858 avec L'Insecte, lorsqu'on les découvre pour la première fois, à la Bibliothèque historique de la Ville de Paris², semblent d'abord particulièrement « lisibles », du moins si on les compare à ceux de Flaubert ou de Joyce par exemple. Les manuscrits appartenant à la phase rédactionnelle sont tous relativement propres, ordonnés, et il arrive même que l'on trouve de longs passages sans ratures et laissés tels quels jusqu'à l'édition finale. Michelet semble en être lui-même conscient, lui qui ne prévoit pas de marges, écrit sur toutes les pages, du haut jusqu'en bas, sans laisser d'espaces vierges, comme s'il ne prévoyait aucun ajout conséquent qui ne pourrait être contenu dans l'espace interlinéaire. Certes il y a bien des ratures dans le manuscrit de Michelet, comme dans tout manuscrit, parfois disséminées sur la page, isolées, mais aussi souvent concentrées dans des passages bien précis, dans des passages difficiles, intenses ou particulièrement originaux. À la lecture de certains passages, un lecteur naïf serait presque tenté de croire que $L a$ Mer correspond à l'idéal de l'œuvre littéraire écrite d'un seul jet par un auteur inspiré. Mais cette image de l'écrivain héroïque, génial, est à n'en pas douter une fiction héritée du romantisme. Bien que lyrique et à bien des égards romantique, Michelet correspond finalement davantage au modèle flaubertien de l'écrivain au travail - il suffit de lire l'ouvrage de Paul Viallaneix, Michelet. Les travaux et les jours, pour s'en convaincre 3 .

Mais il est une autre explication, bien moins glorieuse et bien plus triviale, qui tient moins au caractère de l'auteur qu'au genre de son ouvrage, qui pourrait rendre compte de la relative lisibilité de certains passages du texte de Michelet. Il se trouve en effet que ce chapitre de La Mer qui raconte la migration des harengs de la Baltique à la mer du Nord est largement inspiré de l'article « Hareng », mais aussi, bien que dans une moindre mesure, des articles

1. Il s'agit du premier chapitre du Livre II intitulé « Genèse de la mer », dans Michelet, La Mer, Paris, Édition Jean Borie, coll. « Folio classique», 1983, p. 108-113.

2. Nous nous référerons aux deux volumes disponibles à la Bibliothèque historique de la Ville de Paris du manuscrit intitulé simplement «La Mer», et qui malheureusement n'a pas encore de cote.

3. Paul Viallaneix, Michelet. Les travaux et les jours, 1798-1874, Paris, Gallimard, 1998. 
« Requin » et « Morue », de l'Encyclopédie de Diderot et D'Alembert ${ }^{4}$. Pratique attendue dans un ouvrage de vulgarisation scientifique, la réécriture n'en fait pas moins apparaître un texte sous le texte. Autrement dit, l'avant-texte que constitue notre manuscrit a donc luimême un avant-texte, l'article «Hareng » de l'Encyclopédie, qui d'ailleurs a lui aussi son propre avant-texte puisque l'encyclopédiste, par la nature même de son entreprise totalitaire, est obligé de consulter des sources pour rédiger ses articles. En l'occurrence, c'est un ouvrage du naturaliste Anderson que l'article « Hareng » de l'Encyclopédie cite abondamment. Sans doute cette réalité du processus métatextuel et intertextuel est-elle à différents degrés celle de tout texte littéraire, mais elle s'avère bien plus prégnante et inévitable dans le cas d'un ouvrage de vulgarisation scientifique, et a fortiori de l'encyclopédie, qui n'est que rarement l'œuvre de spécialistes. Or ce qui nous semble faire problème chez Michelet, c'est précisément son rapport ambigu à l'avant-texte : dans un premier temps source de renseignements où il puise des informations, des faits et des données scientifiques, cet avant-texte devient rapidement encombrant et Michelet établit dans un second temps un rapport polémique avec une source qu'il falsifie, réinvente et poétise, pour écrire ce qu'il nommera finalement son grand « poème à la nature ${ }^{5}$ ». En d'autres termes, ce sont moins les passages où il choisit de copier son avanttexte qui nous intéressent, que ceux dans lesquels il décide de s'en écarter, alors même qu'il avait la possibilité de s'en inspirer plus largement. Ou plutôt, c'est le jeu des identités et des différences, ainsi que la nature des écarts, qui doivent avant tout susciter notre intérêt.

Ainsi, l'hypothèse qui guidera notre travail est que la nature profondément hétérogène du manuscrit, à la fois linéaire et sporadiquement miné de ratures, peut s'expliquer par la nature «palimpsestueuse », pour reprendre un terme célèbre de Gérard Genette, du chapitre de Michelet.

Pour que notre analyse soit aussi exhaustive que possible, notre étude génétique du chapitre « Fécondité » sera « horizontale » et non « verticale». Nous nous appuierons sur des documents établis pendant une même phase rédactionnelle de la genèse de $\mathrm{La} \mathrm{Mer}$, afin d'étudier les trois niveaux de texte que constituent l'avant-texte de l'Encyclopédie, le manuscrit de Michelet et le texte définitif. Pour rendre compte des principaux phénomènes génétiques à l'œuvre dans la réécriture de Michelet, nous analyserons d'abord les processus d'amplification et de prolifération (génétiques et thématiques); puis nous montrerons que le texte évolue vers une histoire naturelle qui fait la part belle à la fiction et même à la légende ; enfin nous étudierons la genèse du texte de Michelet comme une réécriture subjective cristallisant bien des obsessions de son auteur.

Le premier chapitre du deuxième Livre intitulé « Genèse de la mer » est tout entier infléchi, d'un point de vue à la fois thématique et génétique, par les phénomènes d'amplification et de prolifération. Ce qui semble en effet fasciner Michelet dans l'épisode migratoire, c'est

\footnotetext{
4. L'influence des auteurs de l'Encyclopédie sur Michelet a été non seulement remarquée et commentée par de nombreux chercheurs, et en particulier par Paule Petitier, lorsqu'elle analyse le double héritage de la Naturphilosophie allemande et de la philosophie des Lumières française dans la pensée de Michelet (La Géographie de Michelet. Territoire et modèles naturels dans les premières auvres de Michelet, Paris, L'Harmattan, 1997), mais surtout revendiquée par Michelet lui-même. Dans l'Histoire de France, il écrivait déjà : « l'Encyclopédie, livre puissant, quoi qu'on ait dit, qui fut bien plus qu'un livre, - la conspiration victorieuse de l'esprit humain ».

5. Michelet désigne par cette expression l'ensemble de son cycle naturaliste composé de L'Oiseau, L'Insecte, La Mer et La Montagne.
} 
ce nombre incroyable de harengs qui, ensemble, se déplacent de la Baltique à la mer du Nord ; nombre qui de surcroît est en constante augmentation tout au long de leur périple, puisque celui-ci a lieu pendant leur période de reproduction. Or nous aimerions montrer que le phénomène génétique de la réécriture tel que la pratique Michelet dans ce chapitre tient lui aussi notamment de l'amplification, de sorte que le processus génétique peut sembler mimétique de la prodigieuse prolifération des harengs 6 .

On remarque ainsi que bien des biffures et que bien des retouches du manuscrit de Michelet concernent non seulement le nombre des harengs migrants, mais aussi la manière de caractériser cet ensemble, son avancée et sa prolifération. On lit par exemple à la page 997 du manuscrit :

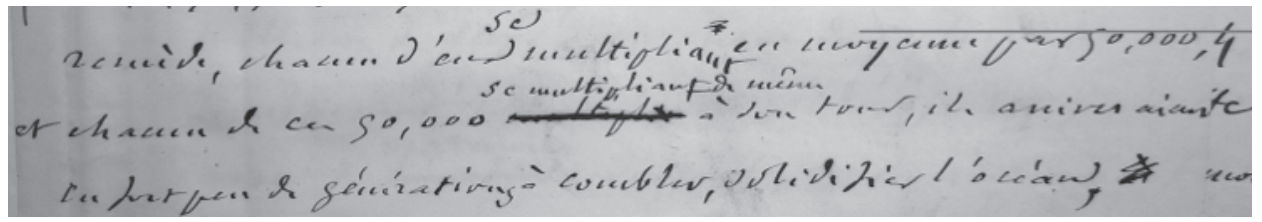

À la première comme à la deuxième ligne, la modification concerne le verbe « multiplier » et à deux reprises, Michelet choisit la forme pronominale - il ajoute d'abord le « se » dans l'espace interlinéaire -, insistant ainsi sur l'idée d'une prolifération rapide, exponentielle et potentiellement infinie, et le participe présent - la terminaison en -ant semble être ajoutée $a$ posteriori, et à la deuxième ligne, «multiplient » est biffé et remplacé par « se multipliant de même »-, qui est une forme virtualisante, caractérisée par sa valeur aspectuelle non accomplie, ce qui permet à Michelet d'insister sur une prolifération continue et en train de se faire. Ce court exemple montre dans un premier temps que Michelet cherche à produire un discours scientifique, mais une confrontation de son texte avec sa source première laisse aussi entrevoir que l'auteur est prêt à lui faire subir une distorsion pour rendre la prolifération plus impressionnante encore : on lit en effet dans la seconde partie de l'article de l'Encyclopédie, «Lorsque les harengs arrivent dans toutes ces mers, ils sont si remplis d'œufs, que l'on peut dire que chaque poisson en amène dix mille avec lui », nombre que Michelet n'hésite pas à multiplier par cinq, et à multiplier à nouveau en ajoutant « et chacun de ces 50000 se multipliant de même », laissant imaginer que cette multiplication peut se poursuivre à l'infini. Il semble que Michelet se soit pris à rêver sur cette expression de l'article : « un nombre si prodigieux, qu'il surpasse tous les nombres connus »; et se soit autorisé, de là, à falsifier le nombre d'œufs, mais aussi, plus loin dans ce même chapitre, le nombre de livres de la laitance des esturgeons qui passe de 1400 dans le manuscrit de Michelet à 14000 dans

6. Précisons que l'hypothèse d'un style mimétique s'accorde à l'une des rares définitions que Michelet a données de son style, définition qui semble à juste titre à Ferdinand Brunot « inquiétante dans son imprécision »: «Le style n'est qu'un mouvement de l'âme » (cité par Ferdinand Brunot dans son Histoire de la langue française des origines à nos jours, nouvelle édition, Paris, Armand Colin, 1966-1979, t. XII, chap. IV, p. 348).

7. Le manuscrit est doublement numéroté : d'abord au crayon, puis chaque chiffre est repassé au stylo, de sorte que deux chiffres apparaissent à chaque page. La numérotation au stylo étant vraisemblablement postérieure, c'est à celle-ci que nous nous référerons ici. 
la version suivante. De même, à la page 100 du manuscrit, après le début, « L'Angleterre y envoie », on déchiffre un premier nombre biffé, après lequel Michelet ajoute « 20 ou 30 mille matelots ».

Les multiples retouches concernant le nombre et la multiplication des harengs dont nous n'avons cité que quelques exemples sont flagrantes, mais il semblerait utile, pour pouvoir les comprendre et les classer, de distinguer au sein de ce même manuscrit différentes campagnes d'écriture auxquelles appartiennent les modifications. Distinguons par exemple deux ratures fort différentes, dans la forme de leur tracé comme dans le moment d'écriture auquel elles appartiennent, de la page 97 du manuscrit :

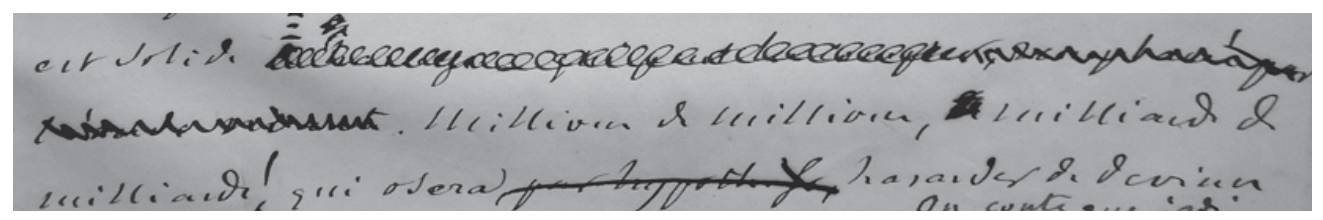

Il apparaît assez nettement que la longue rature en boucles particulièrement denses biffe une phrase entière, qui commence par une majuscule et se termine par un point, après que celle-ci a été écrite et sans lui en substituer une autre ; il s'agit donc plutôt d'une « rayure », le terme renvoyant à l'idée de suppression plus qu'à celle de correction ou de substitution. La seconde, dont le tracé est un simple trait, sec et rapide, supprime dans le fil de l'écriture l'expression « par hypothèse » pour lui préférer le verbe « hasarder », avec lequel l'expression formerait une redondance. À la page $98 \mathrm{du}$ manuscrit, ce sont plusieurs phrases qui sont biffées a posteriori, sans doute lors d'une relecture postérieure à l'écriture du passage, et réécrites dans l'espace interlinéaire :

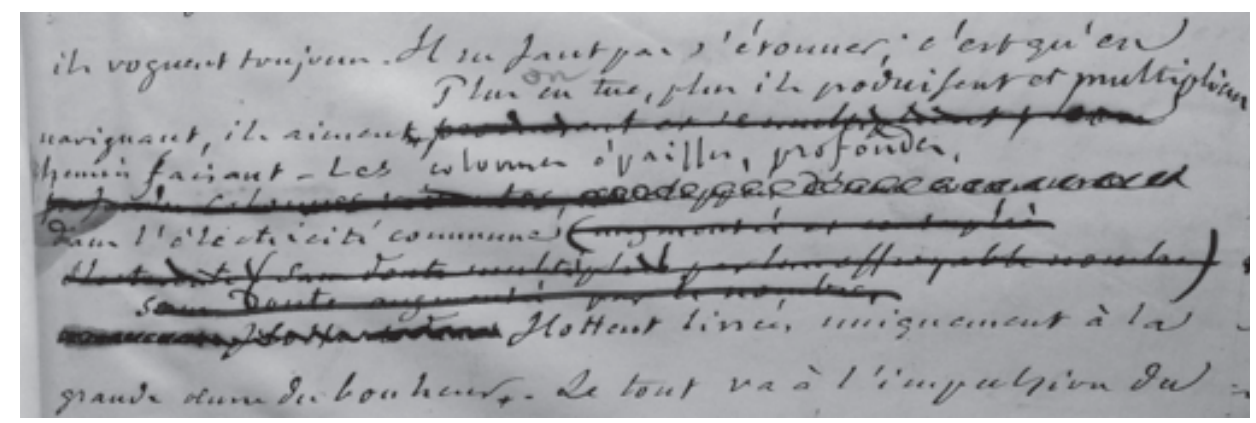

Le segment qui se termine par « ils aiment » se poursuivait d'abord par deux verbes à la troisième personne du pluriel, dont le second est à nouveau « se multiplient ». Michelet supprime plus tard la virgule et commence une nouvelle phrase dans laquelle on aperçoit les mêmes mots que dans la phrase biffée, mais dans une structure comparative - « Plus on en tue, plus ils produisent et multiplient chemin faisant »-plus à même de rendre compte une fois encore de l'idée d'une prolifération incontrôlable. Or dans la suite de l'extrait, 
cette prolifération devient paradoxale puisque l'amplification thématique correspond à une suppression génétique, Michelet procédant dans un premier temps par agrandissement, puis à la relecture, par contraction et suppression. Ainsi, la longue phrase « Les profondes colonnes... » est biffée, coupée, et remplacée dans l'espace interlinéaire par une structure plus dynamique. Puis une longue parenthèse - peut-être ajoutée a posteriori, avant que l'ensemble ne soit supprimé -, contenant une fois encore les termes « augmenté » et « sans doute multiplie par leur effroyable nombre », est rayée et remplacée en dessous, dans l'espace interlinéaire, par «sans doute augmenté par le no » : commençant à écrire une fois de plus « nombre », Michelet s'interrompt pour rayer à nouveau. Après « bonheur », une virgule transformée en point, indique qu'après avoir voulu poursuivre cette longue phrase déjà très retouchée, Michelet choisit de l'interrompre, réduisant finalement sa phrase de huit à deux lignes et demie dans la version finale.

Ce cas de condensation extrême, bien plus flaubertien ${ }^{8}$ que michelétien, nous semble toutefois assez rare. L'analyse génétique des manuscrits de La Mer nous apprend au contraire que Michelet recherche bien plutôt, dans l'élaboration de son texte, un certain type de répétition et de redondance, contrairement à Flaubert qui, on le sait, traquait la moindre répétition et la moindre assonance pour les supprimer. Nombre de passages se construisent ainsi sur des effets de polyptote. En effet, si Michelet supprime l'adjectif «grand» au début de la page $101 \mathrm{du}$

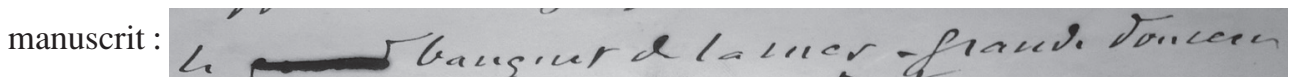

c'est pour éviter une redondance inutile et insignifiante avec « grande douceur ». Au contraire, on lit à la page 99 :

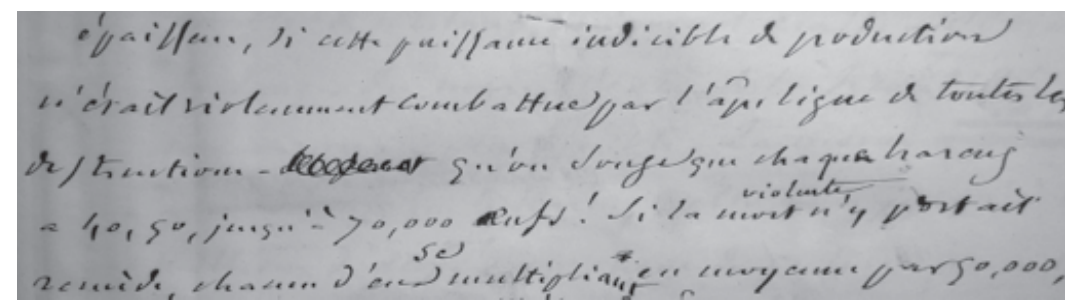

Michelet ajoute a posteriori dans l'espace interlinéaire l'épithète « violente » au-dessus de « mort », créant volontairement un effet de polyptote par dérivation avec l'adverbe « violemment » présent deux lignes plus haut. On retrouve un travail similaire avec les termes «nombre », « nombreux » et « dénombrable », plus loin. À un niveau macrostructural, la recherche de la répétition conduit Michelet à établir une symétrie parfaite entre les deux phrases : «Le merlan s'emplit, se comble de harengs, devient gras », « La morue s'emplit, se comble de harengs, devient grasse ». Au niveau microstructural, aux côtés des effets de polyptote, les effets de synonymie abondent eux aussi : ainsi par exemple, à la page $97 \mathrm{du}$

8. Pierre-Marc de Biasi a montré dans son étude génétique de Saint Julien l' Hospitalier que Flaubert avait coutume de gonfler son texte dans ses premières campagnes d'écriture, multipliant parfois par cinq la taille d'une première version, avant de le réduire considérablement dans les dernières campagnes d'écriture, pour produire une version finale dont le volume est équivalent aux toutes premières versions. 


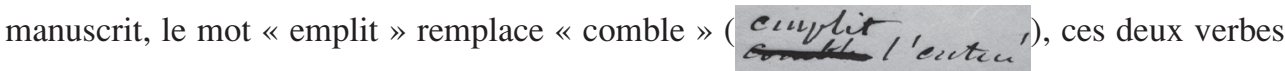
étant récurrents dans tout le chapitre «Fécondité » et déjà très présents dans l'article de l'Encyclopédie, ou encore entre « épaisse » et « grasse », plus loin. Or il se trouve que ces quatre termes étaient tous déjà présents dans l'article de l'Encyclopédie. Des effets similaires sont si nombreux dans le manuscrit qu'il nous est permis de croire que l'effort de Michelet consiste bien à déployer et à amplifier quelques termes cruciaux de sa source qui fixent son attention. Son but n'est donc pas de résumer le contenu de l'article «Hareng » dans un propos dense et efficace, comme on pourrait l'attendre d'un vulgarisateur ordinaire, mais bien plutôt de créer un effet littéraire, en rendant l'idée de prolifération et de germination grâce à des procédés stylistiques.

Cette dernière idée peut ainsi nous permettre de distinguer dans l'écriture de Michelet deux types de prolifération génétique : une «prolifération horizontale », qui concerne les allongements syntaxiques ainsi que les phénomènes de suppression, condensation et dynamisation, et une "prolifération sémantique », qui concerne les effets de redondance, de polyptotes et de synonymie. Or cette double prolifération stylistique, qui nous semble caractéristique de la manière de Michelet, n'est-elle pas tout à fait mimétique de la Fécondité ? L'amplification génétique nous semble en effet imiter à merveille cette puissance génésiaque qu'est la mer, et selon ce rêve mimétique, d'ordre presque cratylien ${ }^{9}$, la genèse du texte serait ainsi à l'image de la genèse de la vie marine. C'est cette prolifération, biologique et stylistique, qui justifie le titre du livre, « Genèse de la mer », ainsi que l'homophonie, largement déployée tout au long de l'ouvrage, entre «mer » et «mère ». Tout porte à croire que pour Michelet cette homophonie, loin d'être anodine, révèle une parenté profonde, féminine, entre les deux puissances génésiaques que sont la femme et la mer. La prolifération stylistique peut-être est là pour rappeler et pour faire sentir ce processus de germination de la mer au lecteur.

Cette notion de prolifération génétique peut enfin nous aider à cerner le rapport ambigu de Michelet à son avant-texte : en effet, si le texte de Michelet se construit d'abord comme un prolongement de l'avant-texte, certains écarts et certaines falsifications mêmes nous permettent d'emblée d'apercevoir un autre rapport, polémique, de l'auteur à sa source principale.

À la lecture du chapitre «Fécondité », il apparaît clairement que Michelet lit et réécrit l'article de l'Encyclopédie avec une, et même avec plusieurs « idées de derrière », et avec certains modèles, littéraire et politique, en tête qui infléchissent, sinon sa lecture, du moins son écriture, et vers lesquels les différentes étapes de la rédaction semblent s'acheminer. Notre hypothèse est que dans la genèse du chapitre s'opère un déplacement de l'Histoire naturelle vers ce que nous pourrions appeler une histoire naturelle, un déplacement de la connaissance vers la fiction, donc.

Afin de rendre compte de ces déplacements, il serait dès lors fort intéressant de réfléchir à ce qui, dans la genèse du texte michelétien, relève de l'exogenèse et de l'endogenèse. Dans le chapitre «Poétique et génétique » de l'ouvrage intitulé Essais de critique génétique, Raymonde Debray Genette définit l'exogenèse comme une « sélection et appropriation

9. À cela près que ce n'est pas le mot, mais l'expression, qui ressemblerait à la chose. 
des sources », et l'endogenèse comme une " production et transformation des états rédactionnels ». Et elle ajoute : «Il existe une sorte particulière de genèse qui montre le chemin de l'endogenèse proprement dite. C'est la réécriture des documents ${ }^{10}$. » Si ce dernier cas concerne sans doute à des degrés divers toute création littéraire, tous genres confondus, il est surtout consubstantiel de tout travail de vulgarisation scientifique. Dans le chapitre «Fécondité », le mouvement dialectique entre le moment de l'exogénétique où la langue se rapporte à quelque chose d'extérieur à elle, à un déjà écrit - en l'occurrence, à l'article de l'Encyclopédie - et le moment endogénétique où l'écriture se recentre sur elle-même et produit du texte sans rien qui ne lui soit extérieur, est porté à son comble, car l'exogénétique se trouve comme incorporé progressivement au texte en cours, prenant la peau, et devenant à son tour, endogénétique. Raymonde Debray Genette poursuit : « le document doit susciter l'envie d'écrire, de réinsérer tel morceau de phrase11 ». Ainsi se dessine, dans la production du texte de Michelet, une intertextualité qui est loin d'être spécifique au genre de l'histoire naturelle, mais qui y trouve une extension et une fonction particulière. Plusieurs termes et expressions de l'article de l'Encyclopédie sont littéralement repris et deviennent les moments fondamentaux du texte de Michelet, tels que « ils sont si remplis d'œufs », « un nombre si prodigieux », « une masse [...] prodigieuse », « voracité des gros poissons » ou encore, à propos de la morue, «goulue et insatiable ». Ne prenons que l'exemple particulièrement frappant d'un ajout de Michelet à la page 102 du manuscrit :

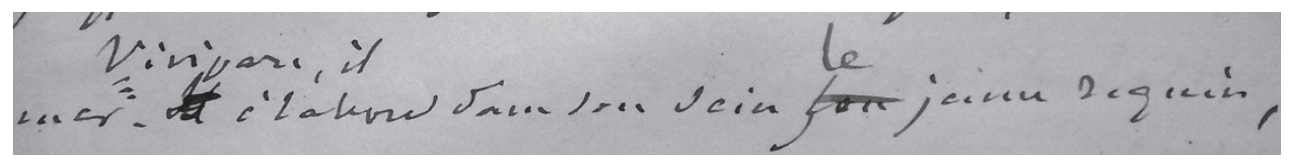

En ajoutant ainsi en début de phrase «Vivipare, il... », Michelet reprend l'adjectif et la structure même d'une phrase de l'article «Requin » de l'Encyclopédie : «Vivipare, le plus grand de tous les chiens de mer... ». Il s'agit donc d'une des formes non explicites de l'intertextualité telle que la définit Gérard Genette dans Palimpsestes - « relation de coprésence entre deux ou plusieurs textes, c'est-à-dire éidétiquement et le plus souvent, par la présence effective d'un texte dans un autre »-, c'est-à-dire le plagiat, en tant qu'emprunt non déclaré, mais encore littéral. À mi-chemin entre l'exogénétique et l'endogénétique, la métatextualité, définie par Gérard Genette comme « relation, dite "de commentaire", qui unit un texte à un autre texte dont il parle, sans nécessairement le citer », est elle aussi très présente dans la production du texte de Michelet. Abordés à partir de ce concept de « métatextualité », les écarts michelétiens par rapport à l'avant-texte de l'Encyclopédie prennent un sens particulier parce qu'ils définissent, en creux, le genre de l'histoire naturelle tel que Michelet le conçoit. Pour reprendre le vocabulaire de Genette, peut-être peut-on considérer que dans le chapitre « Fécondité », l'hypotexte de l'Encyclopédie dérive en hypertexte de manière à la fois massive et, sinon officielle, du moins repérable, pour qui lit ses carnets de travail et son Journal.

10. R. Debray Genette, «Poétique et génétique : le cas Flaubert », dans Essais de critique génétique, Paris, Flammarion, coll. « Textes et Manuscrits », 1979, p. 31.

11. Ibid., p. 32. 
Il est pourtant une des cinq relations transtextuelles repérées par Genette qui pourrait nous induire ici en erreur : l'architextualité, c'est-à-dire le type de discours ou genre littéraire dont relève le texte étudié. La question qui se pose alors est la suivante : comment convientil d'interpréter ces phénomènes de reprise dans un texte d'histoire naturelle et le genre dans lequel ils s'inscrivent leur donne-t-il un sens spécifique?

En effet, si la citation, la reprise plus ou moins littérale, voire le plagiat, font partie de l'horizon d'attente du genre de l'histoire naturelle, les nombreux écarts, déplacements et modifications non négligeables qu'opère Michelet, quittant dès lors le terrain de la science pour celui de la philosophie, de la morale, ou de la fiction, ne sont pas consubstantiels au genre. La preuve la plus flagrante d'un tel choix est la suppression, à la page $102 \mathrm{du}$ manuscrit, d'un très long paragraphe largement inspiré de ses notes de travail et dont Michelet avait déjà commencé à retrancher la plupart des références et des données scientifiques, avant de le supprimer définitivement dans la version suivante, au profit de la narration. Le passage commence ainsi : "C'est depuis cent ans seulement, je veux dire depuis Linné qu'on a commencé d'entrevoir l'épouvantable richesse et végétale et animale qui fleurit» :

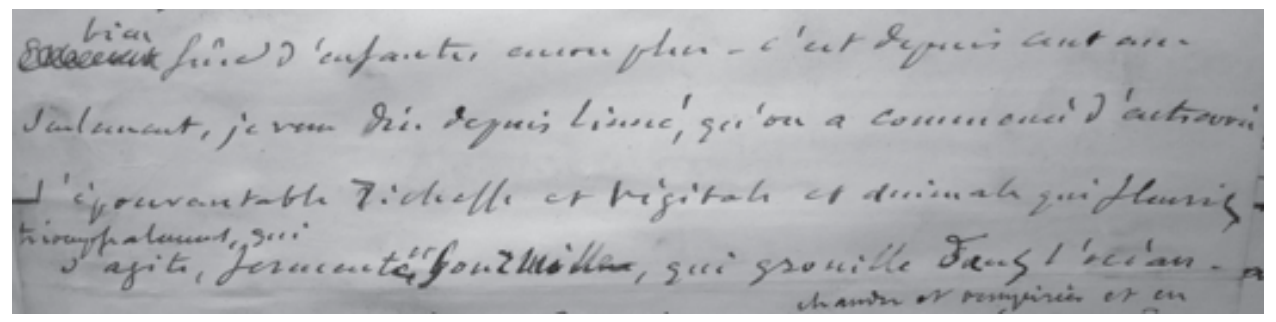

Et Michelet d'ajouter, comme pris par un besoin d'expansion, d'amplification, d'allitération et d'assonance, dans l'espace interlinéaire puis linéaire : « triomphalement qui s'agite, fermente et fourmille, qui grouille dans l'océan ». S'ensuivent plusieurs chiffres et remarques sur les espèces sous-marines, et surtout une référence à Darwin, extrêmement précieuse puisque les commentateurs n'ont de cesse de s'étonner que Michelet ne connaisse pas ou n'ait pas lu Darwin, tandis que l'absence du naturaliste anglais apparaît bien plutôt, à la lumière de l'étude génétique, comme un choix idéologique volontaire. C'est que Michelet n'hésite pas à favoriser le récit et la narration, retranchant ou distordant certains faits. Ainsi dès l'ouverture du chapitre, où il écrit « Dans la nuit de la Saint-Jean (du 24 au 25 juin), cinq minutes après minuit, la grande pêche du hareng s'ouvre dans les mers du Nord », inventant cette fois un excès de précision, par rapport à l'article de l'Encyclopédie dans lequel on lisait simplement « à peu près au commencement de Juin », pour faire voyager ses harengs un jour de fête, puisqu'il s'agit bien, pour Michelet, d'une " grande fête d'amour », d'une fête orgiaque. Par bien des procédés rhétoriques et stylistiques, Michelet fait de ce jour une fête, mais aussi une légende. Pour donner à son conte un air de vérité, il invente des paroles, insérant dans son texte des passages de discours direct : p. 97, il biffe un début de

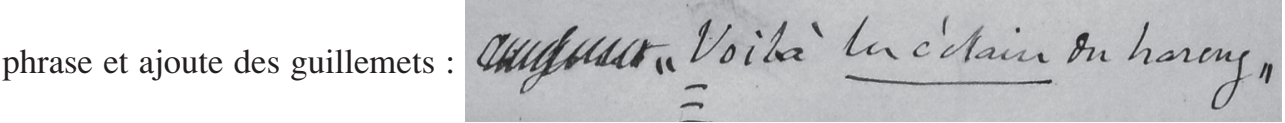


et, plus loin, avant d'écrire «C'est (disaient les Flamands) comme si nos dunes se mettaient à voguer », Michelet retravaille à plusieurs reprises une phrase plus longue et détaillée :

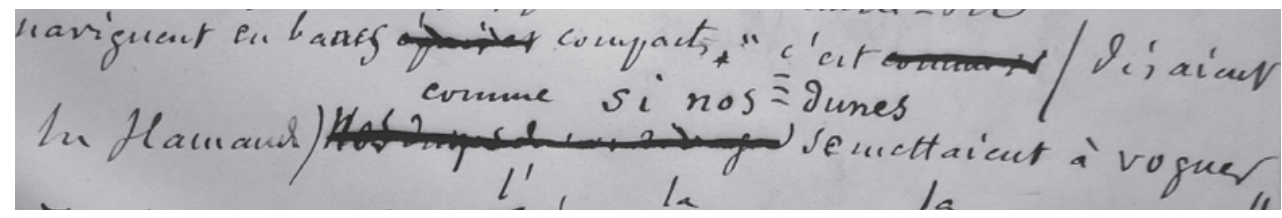

Autant de ratures dans un discours direct nous montrent qu'il s'agit d'un discours inventé, car si Michelet se contentait de recopier des moments de l'article de l'Encyclopédie, il n'aurait pas besoin de biffer et de réécrire. Par ce procédé, Michelet cherche à donner ce que nous pourrions appeler un « effet de légende » et à donner corps à son récit. Tout aussi signifiante est la correction qui, un peu plus loin dans cette même page, remplace un début de phrase commençant par «Quand... » par un « On conte que jadis près du Havre », faisant le choix du conte ou de la légende, au détriment de l'histoire :

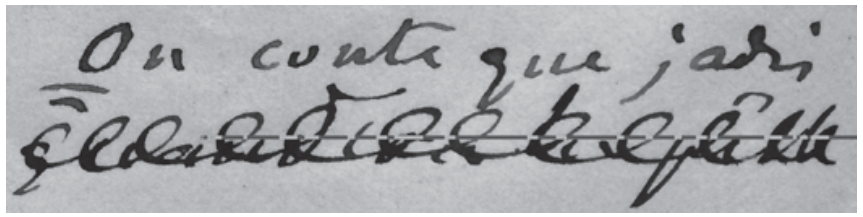

De son expérience d'historien, Michelet garde aussi le souffle épique. Les traits caractéristiques du genre de l'épopée se retrouvent en effet dans tout notre extrait et constituent à n'en pas douter l'un des principaux écarts de l'écrivain face à sa source mais aussi face au genre de l'histoire naturelle. Sont épiques les dénominations telles que « les légions de harengs » (p. 108), et tous les procédés qui tendent à représenter les bancs des harengs comme un peuple - la répétition du mot « ensemble » par exemple dans ces quelques lignes : «Ils montent, ils montent tous d'ensemble, pas un ne reste en arrière. La sociabilité est la loi de cette race ; on ne les voit jamais qu'ensemble. Ensemble ils vivent ensevelis aux ténébreuses profondeurs ; ensemble ils viennent au printemps prendre leur petite part du bonheur universel, voir le jour, jouir et mourir » (p. 107). Le terme « ensemble » est et en anaphore, et en épiphore, et en anadiplose : il scande littéralement le texte, l'envahit. Ainsi le surgissement des harengs, ou plutôt des « légions du hareng », a-t-il l'allure d'une insurrection, et d'une insurrection particulièrement menaçante car elle risquerait, si elle ne rencontrait aucune opposition, de rendre la mer solide.

On comprend ainsi aisément que l'arrivée du requin soit un passage paradoxal, source d'angoisse et promesse d'apaisement. C'est le passage le plus bouleversé, le plus raturé et retouché du texte. Il est écrit de plus en plus mal, sans doute de plus en plus vite. La première phrase n'a jamais changé pourtant, avec son étonnant adjectif substantivé : «Il faut que la nature s'invente un suprême dévorateur. » Michelet cherche son qualificatif « avide », « avare », alors qu'il cherche peut-être le verbe « avale », qui scandera le texte, préféré à « absorbe », biffé juste avant - et écrit finalement « Monstre secourable et terrible 
qui consume ce monde », puis choisit l'hypallage du « flot invincible » et la métaphore de lame qu'il préfère à celle du feu, et écrit : «Monstre secourable et terrible qui coupe ce flot invincible de fécondité renaissante » (p. 101).

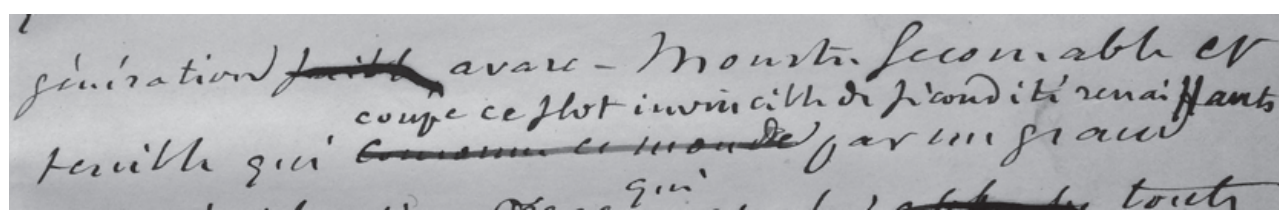

Et la monstruosité de prendre, comme souvent chez Michelet, un sens politique et idéologique, lorsque, face au "peuple» des harengs, le requin fait figure de monarque sanguinaire lorsqu'il écrit « le jeune requin, son héritier féodal, qui naît terrible et tout armé ».

Mais il se profile aussi dans cette représentation du requin une idée bien plus originale que la simple « historicisation » de l'animal. En effet, l'angoisse qui saisit Michelet face à la fatalité de l'enfantement et à l'inévitable surpopulation qui en découle, le conduit à formuler une thèse tout à fait nouvelle dans le discours naturaliste, qui consiste à valoriser la destruction et donc la mort. Michelet nous semble avoir une vision tout à fait originale lorsqu'il accorde au requin, dans la dernière partie du chapitre, une fonction bénéfique, salvatrice et salutaire, et c'est peut-être pour cela que ce passage est nettement plus raturé que le reste du chapitre. Le requin apparaît dans le début du passage que nous citions plus haut comme un deus ex machina, qui crée la surprise tout en donnant un nouveau souffle à l'action, empêchant la solidification de la mer, qui apparaissait pourtant comme une fatalité. Facteur de destruction et animal providentiel, le requin devient ainsi un animal hautement ambivalent, dont l'ambiguïté se trouve condensée dans la formule oxymorique "monstre secourable et terrible ». Peut-être Michelet est-il l'un des premiers à accorder une valeur si positive aux prédateurs, et à pousser si loin l'idée d'un équilibre des forces, bénéfiques et maléfiques, dans la nature. Le plus grand prédateur de la mer symbolise ainsi ce « mal nécessaire » dont il est souvent question en philosophie politique, et même - si la virilisation extrême du requin peut nous autoriser ce jeu de mot - un «mâle nécessaire ». Remarquons, dans ce même ordre d'idée, que les harengs suivent une trajectoire symbolique inverse, et passent du pôle très positif de la fécondité, à celui, négatif, de la dangerosité. Et ce sont ces axes interprétatifs nouveaux, mettant au jour l'équivocité d'une nature toujours énigmatique, et signalant ce faisant les limites de toute interprétation humaine des phénomènes naturels, qui nous conduisent à parler d'une histoire naturelle subjective.

L'analyse génétique du chapitre « Fécondité » montre en effet clairement que le texte de Michelet s'achemine vers une histoire naturelle profondément subjective, avec tous les paradoxes et toutes les tensions que l'expression comporte, dans une écriture qui cristallise bien des obsessions de son auteur.

Et c'est précisément dans l'invention d'un anthropomorphisme très particulier que réside à nos yeux l'originalité de l'histoire naturelle michelétienne. Or cet anthropomorphisme non naï, spécifiquement michelétien, constitue justement le point de rupture de Michelet avec l'article de l'Encyclopédie. Ainsi, dans le chapitre «Fécondité », l'anthropomorphisme est 
nettement articulé à la question des genres, masculin et féminin, mais aussi, en creux, humain et animal. Par exemple, le traitement du terme « Fécondité » au cours du chapitre sert de pivot au jeu homonymique entre «mer » et «mère » et témoigne d'une porosité des genres, masculin et féminin, dans l'élément marin tel que Michelet l'imagine. Les manuscrits du chapitre nous apprennent que Michelet a hésité à appeler son chapitre « Fécondité de la mer », avant de l'appeler simplement «Fécondité », masquant ainsi l'origine et l'appartenance, masculine, féminine ou anonyme, de l'effusion séminale dans laquelle baigne tout le chapitre. Le choix du seul terme «Fécondité », permet de n'insister que sur le procès et sur la substance. Or la substance semble être l'une des composantes principales de l'érotisme michelétien tel qu'il se déploie dans ce chapitre, de sorte que l'anthropomorphisme passe par l'érotisation de la mer et de la vie sous-marine tout entière. Plusieurs corrections de Michelet corroborent l'hypothèse d'une progressive érotisation; ainsi, à la page 97 du manuscrit, au moment de l'insurrection des harengs,

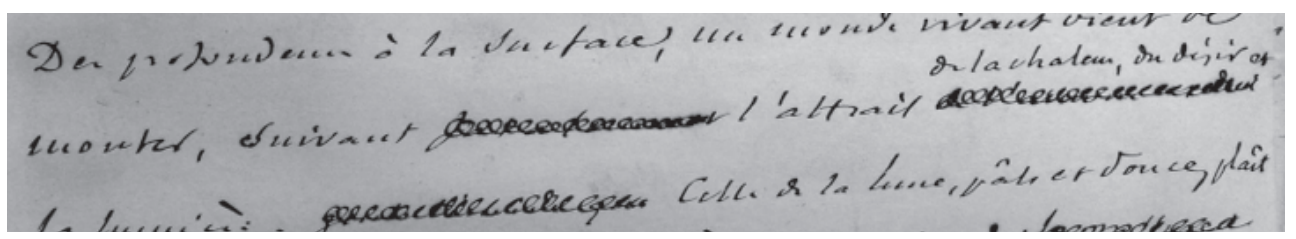

dans la phrase commençant par « Des profondeurs à la surface, un monde vivant vient de monter... », Michelet substitue a posteriori « de la chaleur, du désir », amorçant ainsi dès l'ouverture du chapitre ce que nous pourrions appeler un anthropomorphisme érotique, qui se doublera plus loin d'un anthropomorphisme émotionnel ou psychologique qui culmine dans les termes d' «amour » ou de «bonheur» appliqués aux harengs. De même, dans la phrase « Dans ce monde qui ne connaît pas l'union fixe, le plaisir est une aventure, l'amour une navigation », «l'union fixe » vient remplacer un terme bien plus concret, « l'accouplement » :

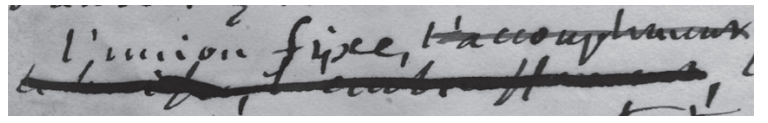

Dans la phrase suivante, Michelet avait d'abord écrit « Sur toute la route ils épanchent l'espoir des torrents », puis il biffe le mot «espoir» pour écrire «Sur toute la route ils épanchent des torrents de fécondité », avant de biffer puis de réécrire, dans une encre maintenant plus claire, le terme « fécondité » :

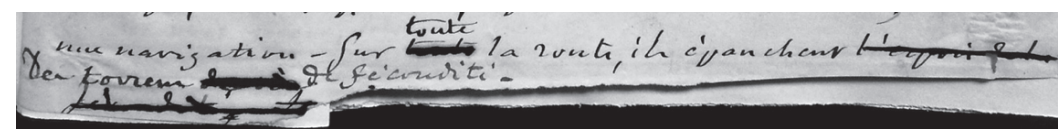

Une telle hésitation peut venir du fait que l'expression «torrents de fécondité » ne va pas de soi et n'est pas naturelle, pas même à Michelet ; c'est une construction qui mêle dans 
l'imaginaire masculin et féminin, puisque le torrent connote traditionnellement la sexualité masculine, tandis que la fécondité est la capacité dont jouissent les femelles d'assurer la reproduction de l'espèce. La mer deviendrait ainsi, sous la plume de Michelet, comme un double matériel de lui-même lorsqu'il écrivait : «Je suis un homme complet ayant les deux sexes de l'esprit. »

En effet, si l'effusion séminale des «torrents de fécondité » est caractéristique de l'érotisme michelétien, en tant qu'elle suscite chez lui à la fois fascination, répulsion et même angoisse, c'est aussi parce qu'elle répond à un désir et à un idéal qui infléchit toute sa vision de la nature : le désir d'unité. Cet idéal michelétien est donc palpable tout au long du chapitre, sans que pour autant le texte n'en devienne trop abstrait, puisque ce qui intéresse au contraire Michelet c'est la matière même de cette unité dont il tente de saisir la substance propre. Ainsi, les différentes étapes du chapitre - l'aventure amoureuse des harengs, le surgissement de l'esturgeon, de la morue, puis du requin - correspondent aux différents états de la mer qui change d'aspect au fur et à mesure - virginale d'abord, puis visqueuse, grasse, et enfin solide. Le traitement de l'adjectif « épais » dans la production du texte de Michelet nous semble significatif de son effort pour transcrire le plus fidèlement possible la substance de la mer. D'abord, le mot est appliqué aux légions de harengs pour rendre compte de leur densité (p. 97) :

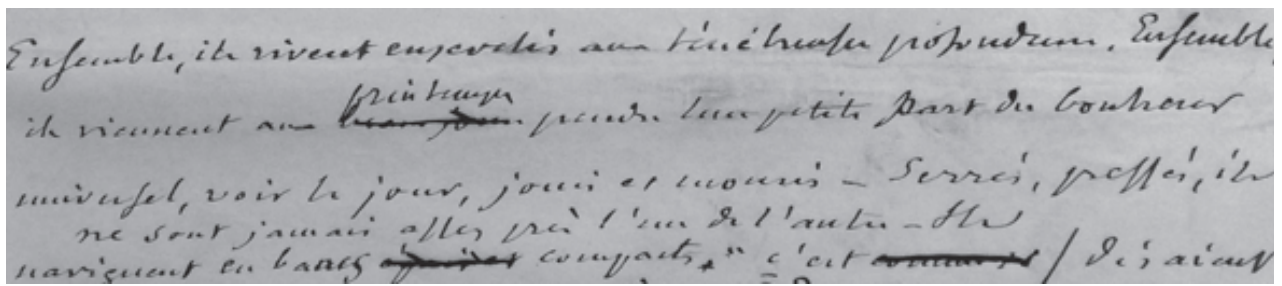

Dans les phrases «Ensemble, ils vivent ensevelis aux ténébreuses profondeurs ; ensemble ils viennent au printemps prendre leur petite part du bonheur universel, voir le jour, jouir, et mourir », l'anaphore de « ensemble » soutenue par l'homophonie avec « ensevelir », puis celle de « jour », « jouir » et «mourir », après les deux participes passés «serrés, pressés », Michelet ajoute dans l'espace interlinéaire «ils ne sont jamais assez près l'un de l'autre », puis «Ils naviguent en bancs épais et compacts ». Pourquoi biffe-t-il « épais » a posteriori, refusant une nouvelle redondance alors que le passage entier joue sur les répétitions phoniques et sémantiques? Le travail du texte dans les pages suivantes du manuscrit nous aide à répondre : Michelet semble vouloir donner un sens et une extension bien particulière à l'adjectif, il ne veut pas que celui-ci s'applique aux poissons uniquement, mais qu'il reste associé à la matière, à la viscosité de la mer au moment précis où le poisson et elle ne font plus qu'un. En témoigne l'ajout, à la page 99 du manuscrit, dans l'espace interlinéaire après « La baleine et les cétacés ne dédaignent pas ce gibier, ils le fuient », de l'expression « ils plongent dans les bancs, entrent dans l'épaisseur vivante » : 


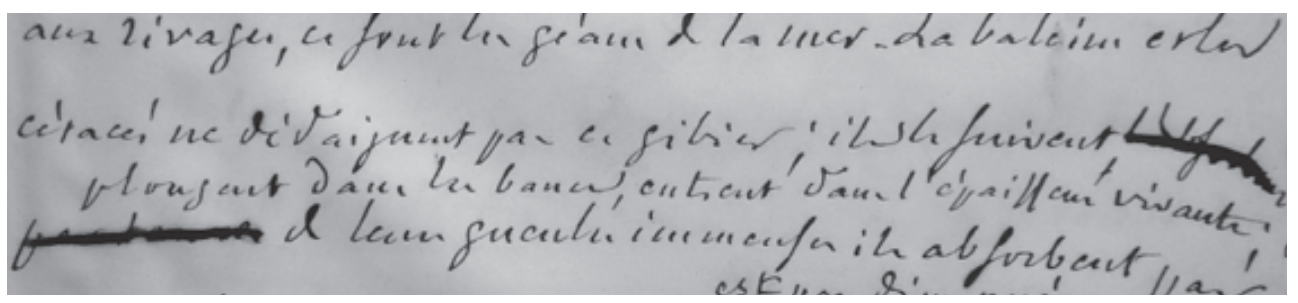

L'apparition du substantif « épaisseur » ne pouvait avoir lieu qu'après l'effusion séminale, et non avant, à l'ouverture du chapitre. L'expression surprenante « entrent dans l'épaisseur vivante » est à la fois un éloignement du contexte précis, une plus grande abstraction, et une insistance plus appuyée sur la matière même, une proximité accrue avec le sensible. On retrouve cette même articulation du concret et de l'abstrait, très prégnante dans les ouvrages naturalistes de Michelet, dans l'expression « les moindres poissons avalent le frai et les œufs du hareng, se gorgent de laite, mangent l'avenir », et à la page 101 du manuscrit, lorsque Michelet ajoute dans l'espace interlinéaire, après « Bonheur infini », « pour lui de trouver là concentrée la substance », et à la ligne « de mordre en chair pleine, en viande épaisse », c'est-à-dire :

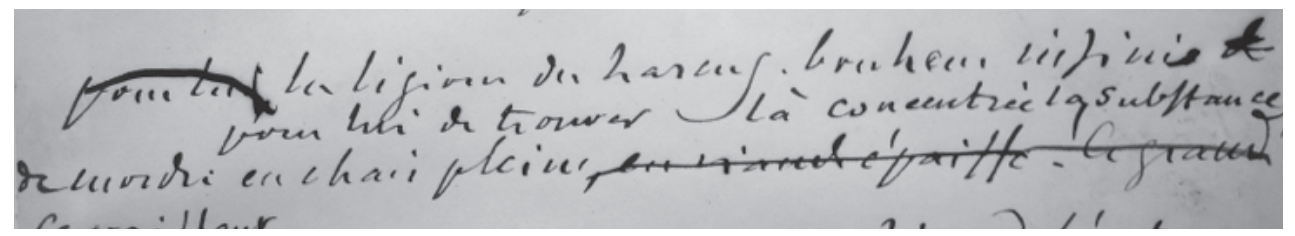

Dans tous ces exemples, la substance maritime de la saison des amours est liée à l'oralité et à l'absorption, et pour ces deux phénomènes, Michelet s'emploie aussi à trouver le mot juste, d'où la récurrence tout au long du chapitre des verbes « avaler », « absorber », « manger » et des substantifs, verbes ou adjectifs substantivés « mangeur », « dévorateur », " goulue », « glouton », qui rejoignent chez Michelet à la fois un idéal d'oralité virile - figure du requin en monarque glouton - et un idéal de purification par la destruction - les requins répondent à un surplus de fécondité -, qui s'exprime à maintes reprises dans ses écrits naturalistes. Ces deux réseaux sémantiques s'unifient finalement difficilement, dans la dernière page très retouchée du manuscrit, qui subira beaucoup de modifications, dans l'image finale de la «mer absorbante ». Une longue phrase très retravaillée sur la « molle vie absorbante du poisson » dans les « eaux épaisses » disparaîtra finalement de l'édition finale, mais un morceau de papier blanc collé en bas de la page empêche de lire la phrase entièrement. Puis l'image de la «mer absorbante » se prépare, dans cette phrase, « La mer coule indéfiniment. Indéfiniment absorbe, cette mer nageante et vive qu'on appelle poisson », avançant encore par répétitions, dans une structure en chiasme découpé en deux phrases, tout à fait mimétique, comme l'hypallage de la «mer nageante », de l'image d'une mer-animale telle que l'esquisse Michelet (p. 105) : 


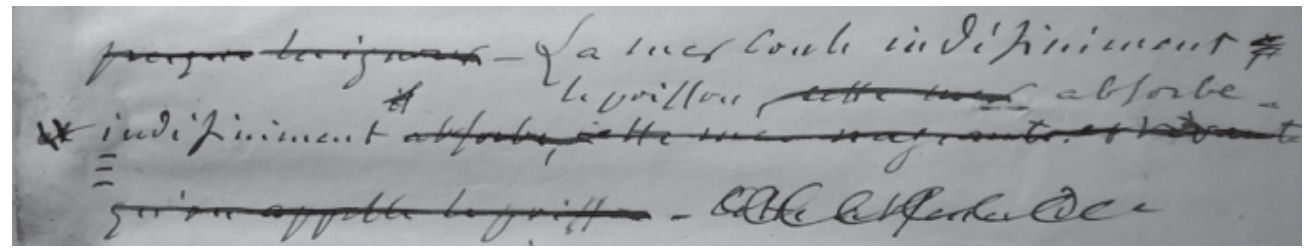

Au-dessus de la rature, Michelet tente de réécrire sa phrase en utilisant à nouveau ces mêmes mots, et on lit «le poisson, eette mer, absorbe », sans parvenir toutefois à une version qui le satisfasse et la phrase restera incomplète. Les mêmes hésitations se poursuivent dans la phrase suivante où Michelet remplace « absorbe » par « avale » :

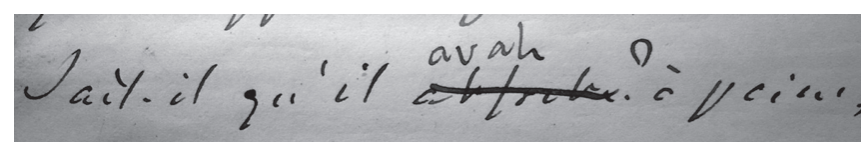

Enfin, la comparaison qui suit, « La nourriture même est comme un lait qui vient à lui », prépare une nouvelle expression de l'unité, de la concentration, de la totalité et du microcosme, que Michelet met en valeur en traçant un trait rouge dans la marge : « Tout est sperme et tout est œuf. C'est dans la mer, une autre mer. »

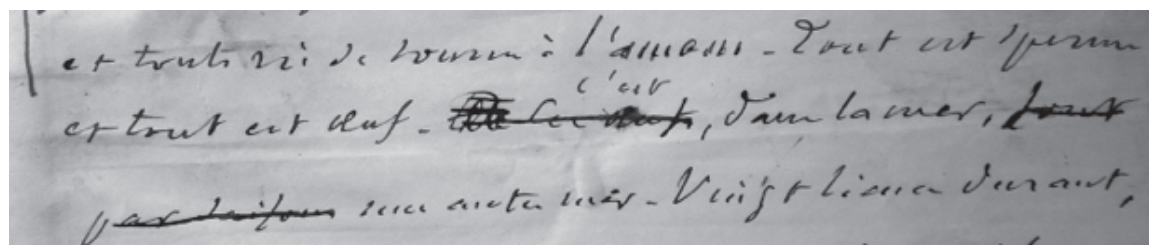

Ces derniers exemples nous semblent significatifs de l'histoire naturelle subjective vers laquelle Michelet s'achemine dans ses manuscrits. Il s'y manifeste une lecture typiquement romantique des phénomènes naturels, tout particulièrement palpable dans le désir d'unité, dans le rêve d'une forme commune et primordiale des êtres et des règnes, et dans l'insistance sur le processus même d'enfantement et de germination de la nature. Si l'histoire naturelle de Michelet est subjective, c'est aussi et surtout parce que son écriture naturaliste, loin de n'être qu'une réécriture d'un déjà écrit, cristallise bien des obsessions qui lui sont propres, telles que l'angoisse de l'excès et du trop-plein, l'épaisseur du monde et des substances à la fois fascinantes et répulsives, dessinant ce faisant un érotisme spécifiquement michelétien.

Si le travail de naturaliste de Michelet semble d'abord être celui d'un compilateur et d'un vulgarisateur, le chapitre "Fécondité » est bien plus qu'une réécriture de l'article de l'Encyclopédie. Michelet se nourrit de son avant-texte pour écrire un tout autre texte. Ainsi, si l'intertextualité et la métatextualité telles que les a définies Gérard Genette font partie intégrante de la production du texte, les phénomènes intertextuels, présents dans toute œuvre littéraire et très étudiés par la critique, ont une importance presque inversement 
IN TERTEXT U A L T É ET HISTOIRE NAT URELLE

$\begin{array}{lllll}V & A & R & I & A\end{array}$

proportionnelle dans les ouvrages d'histoire naturelle de Michelet, où ce sont les écarts, les moments d'infidélité qui sont les plus précieux. Là où l'article scientifique décrit et explique, le texte de Michelet interprète et donne à vivre. Là où l'article scientifique est dense, d'une progression linéaire et sans redite, le texte de Michelet déploie des images fortes et des motifs obsessionnels, il avance par à-coups, par répétitions et par redondances, et se construit autour de mots pivots, qui sont dédoublés, répétés, dérivés à l'infini. L'esthétique michelétienne est à la fois une esthétique du polyptote et de l'hypallage, de l'amplification et de la concentration, qui s'exprime dans une écriture du flux et du reflux, une écriture de l'éternel retour, du désir, ou de la mer.

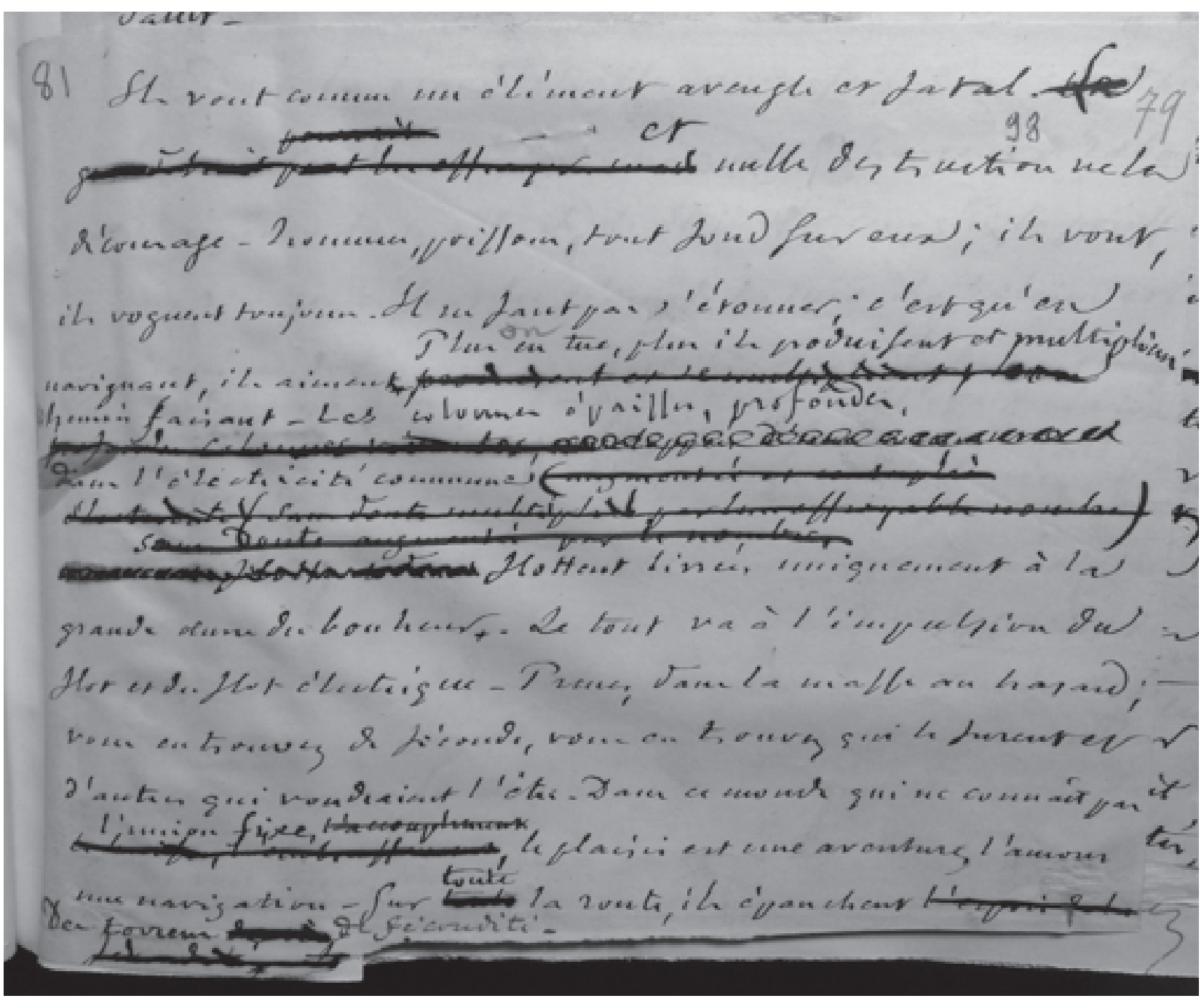

Jules Michelet, manuscrit de La Mer, Livre II, chap. I, f 98 (Bibliothèque historique de la Ville de Paris)

201 


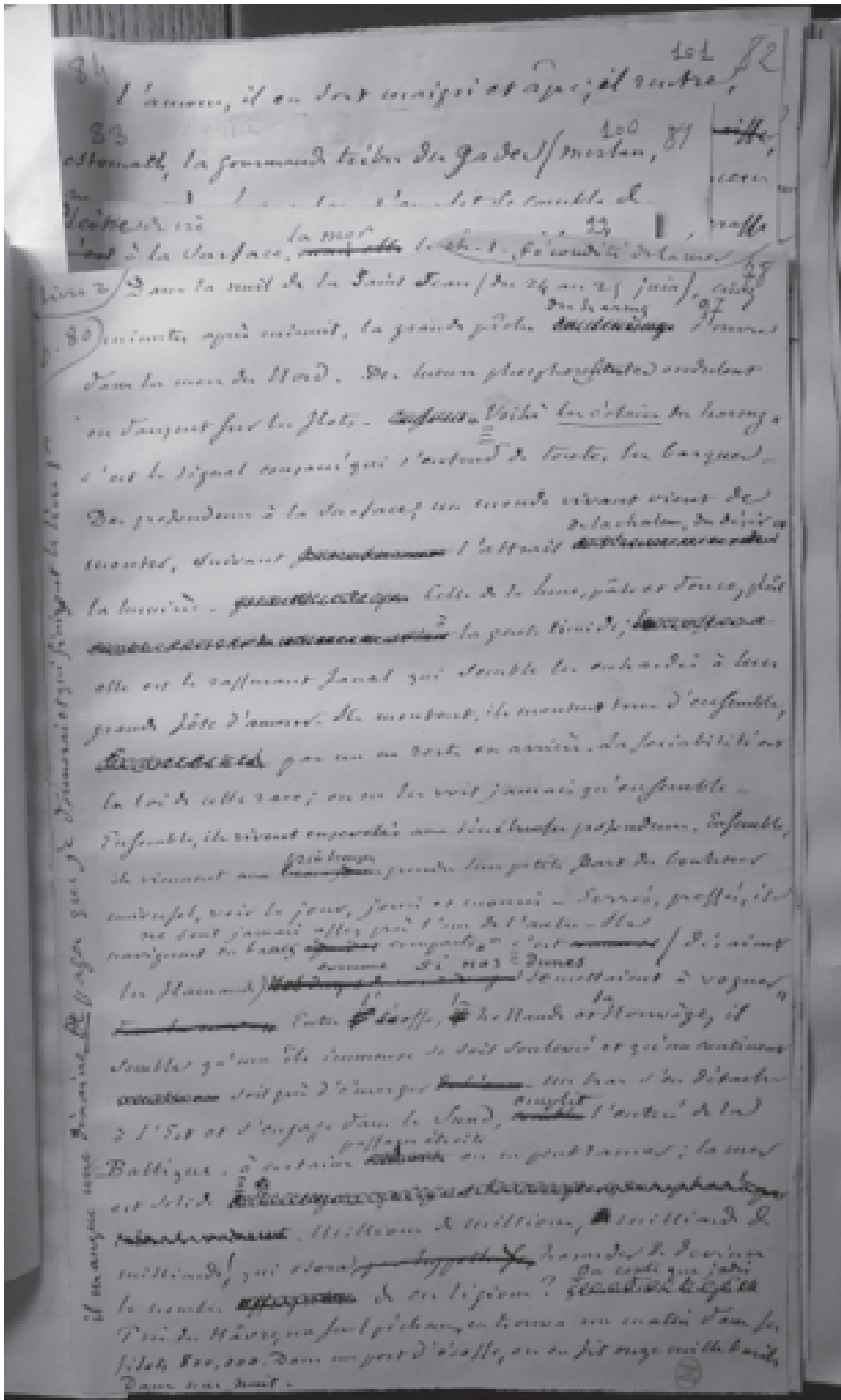


Élisabeth Plas, née en 1988, est entrée à l'ENS en 2009. Son mémoire de Master 1, dirigé par Didier Philippot, portait sur les métaphores et métamorphoses de la femme-panthère chez Balzac et Barbey d'Aurevilly et son mémoire de Master 2, dirigé par Paolo Tortonese, sur l'anthropomorphisme dans le cycle naturaliste de Michelet. Elle publiera prochainement, dans un ouvrage collectif dirigé par Alain Schaffner, un article sur l'anthropomorphisme et l'empathie chez Michelet.

elisabeth.plas@ens.fr

Résumés étude génétique du chapitre "Fécondité " de La Mer de Michelet

L'intertextualité, qui traverse sans doute la production de tout texte littéraire, est une pratique courante et même inévitable dans un ouvrage de vulgarisation scientifique, tel que La Mer de Michelet, dont de nombreux passages ont pour avant-texte l'Encyclopédie de Diderot et D'Alembert. Cette étude génétique d'un chapitre du Livre «Genèse de la mer » s'attachera moins aux phénomènes de reprises et de citations, comme d'autres ont pu le faire, qu'aux écarts et au jeu de différences, qui révèlent un rapport polémique de Michelet à sa source et permettent d'identifier - grâce à une étude comparative des avant-textes, de la phase rédactionnelle du manuscrit et du texte final - à la fois une conception nouvelle de l'histoire naturelle et une pratique d'écriture, répétitive et insistante, spécifiquement michelétiennes.

Intertextuality, which probably runs through all literary texts' production, is a common and even inevitable practice in a scientific work aimed at the general public such as Michelet's La Mer, many passages of which have as "avant-texte" Diderot and D'Alembert's Encyclopédie. This genetic study of the chapter "Genèse de la mer" focuses less on the presence of repetitions and quotations, as others have already done, than on the differences: they reveal Michelet's polemical relationship to his source and allow us - through a comparative study of the avanttextes, the writing phase of the manuscript and the final text - to identify both a new conception of Natural History and a writing practice, repetitive and insistent, specific to Michelet.

Die Intertextualität, die ohne Zweifel alle literarische Produktion durchzieht, ist eine geläufige Praxis und schlicht unvermeidbar bei einem populärwissenschaftlichen Werk wie Michelets $L a$ Mer, zu dessen „Avant-texte“ zahlreiche Passagen aus der Encyclopédie Diderots und D’Alemberts gehören. Die vorliegende genetische Studie zu einem Kapitel des Buches „Genèse de la mer", interessiert sich weniger für Phänomene der Wiederaufnahme und des Zitats - wie dies von anderen schon gezeigt wurde - als für das Spiel mit Brüchen und Abweichungen, die einen polemischen Dialog Michelets mit seiner Quelle offenbaren und es erlauben - dank einer vergleichenden Studie der „Avant-textes“, der Schreibphase des Manuskriptes, sowie des fertigen Textes - zugleich eine neue Konzeption von Naturgeschichte und eine repetitiv-beharrliche, für Michelet charakteristische, Schreibpraxis zu identifizieren.
La intertextualidad, que atraviesa sin duda la producción de todo texto literario, constituye una práctica corriente, e incluso inevitable, en una obra de divulgación científica, como El mar de Michelet, muchos de cuyos pasajes tienen como pre-texto $L a$ Enciclopedia de Diderot y D’Alembert. Este estudio genético de un capítulo del libro, "Génesis del mar", tratará, no tanto de los fenómenos de repetición y cita, que ya otros han podido realizar, como de los distanciamientos y el juego de diferencias, que ponen de manifiesto una relación polémica de Michelet con su fuente y permiten identificar -gracias a un estudio comparativo de los pre-textos, de la fase redaccional del manuscrito y del texto finaluna concepción nueva de la historia natural y, al mismo tiempo, una práctica de la escritura, repetitiva e insistente, específica del autor.

L'intertestualità, che senza dubbio concerne la produzione di qualunque testo letterario, è una pratica corrente e anche inevitabile in un'opera di popolarizzazione scientifica come $\mathrm{La} \mathrm{Mer}$ di Michelet, numerosi passaggi della quale hanno come avantesto l'Encyclopédie di Diderot e D'Alembert. Questo studio genetico di un capitolo del libro "Genèse de la mer" verterà meno sui fenomeni di ripresa e citazione, già analizzati da altri studiosi, che sugli scarti e le differenze che mostrano un rapporto polemico di Michelet con la sua fonte e permettono di identificare - grazie a uno studio comparativo degli avantesti, della fase redazionale del manoscritto e del testo finale - sia una nuova concezione della storia naturale sia una pratica di scrittura, ripetitiva e insistente, specifiche di Michelet.

A intertextualidade, que percorre a produção de qualquer texto literário, é uma prática corrente e mesmo inevitável numa obra de vulgarização científica, como La Mer de Michelet, de que numerosas passagens têm por ante-texto a Enciclopédia de Diderot e D'Alembert. Este estudo genético de um capítulo do livro, "Génese do mar", preocupa-se menos com fenómenos de cópia e citação que com os desvios e as diferenciações, que revelam a relação polémica de Michelet com a sua fonte e permitem identificar - graças a um estudo comparativo dos antetextos, da fase redaccional do manuscrito e do texto final - tanto uma concepção nova da história natural como uma prática de escrita, repetitiva e insistente, muito própria de Michelet. 